Research paper

\title{
ChIP: An early activation protocol for isolated blunt chest injury improves outcomes, a retrospective cohort study
}

\author{
Kate Curtis ${ }^{\mathrm{a}, \mathrm{b}, \mathrm{c}, \mathrm{d}, *, \text {, Stephen E. Asha }}{ }^{\mathrm{c}, \mathrm{e}}$, Annalise Unsworth ${ }^{\mathrm{c}}$, Mary Lam ${ }^{\mathrm{f}}$, \\ Helen Goldsmith ${ }^{\mathrm{b}}$, Mary Langcake ${ }^{\mathrm{b}}$, Donovan Dwyer $^{\mathrm{e}}$ \\ a Sydney Nursing School, University of Sydney, Mallett St, Camperdown, NSW, Australia \\ b Trauma Service, St George Hospital, Gray Street, Kogarah, NSW, Australia \\ ' St George Clinical School, Faculty of Medicine, University of New South Wales, High Street, Kensington, NSW, Australia \\ d The George Institute for Global Health, Bridge Street, Sydney, NSW, Australia \\ e Department of Emergency Medicine, St George Hospital, Gray Street, Kogarah, NSW, Australia \\ ${ }^{\mathrm{f}}$ Faculty of Health Sciences, University of Sydney, East Street, Lidcombe, NSW, Australia
}

\section{A R T I C L E I N F O}

\section{Article history:}

Received 22 April 2016

Received in revised form 9 June 2016

Accepted 12 June 2016

\section{Keywords:}

Injury

Rib fracture

Pain management

Clinical guideline

Emergency

Nursing

\begin{abstract}
A B S T R A C T
Background: Blunt chest injuries not treated in a timely manner with sufficient analgesia, physiotherapy and respiratory support are associated with increased morbidity and mortality. The aim of the study was to determine the impact of a blunt chest injury early activation protocol (ChIP) on patient and hospital outcomes.

Methods: In this pre-post cohort study, the outcomes of patients with blunt chest injury who received ChIP were compared against those who did not. Data including injury severity, patient outcomes, hospital treatments and comorbidites were extracted from medical records. The primary outcome was pneumonia. Secondary outcomes evaluated health service delivery. Logistic and multiple regressions were used to adjust for potential confounding variables.

Results: 546 patients were included, 273 in the before-ChIP cohort and 273 in the after-ChIP cohort. The incidence of pneumonia following the introduction of ChIP was reduced by $4.8 \%(95 \% \mathrm{CI} 0.5-9.2, \mathrm{p}=0.03)$. In the after-ChIP cohort, more patients received a pain team review ( $32 \%$ vs. $13 \%, \mathrm{p}<0.001$ ), physiotherapy ( $93 \%$ vs. 86\%, p = 0.005) and trauma team review (95\% vs. 39\%, p<0.001). There was no difference in length of stay $(\mathrm{p}=0.50)$.

Conclusions: ChIP improved the delivery of healthcare services and reduced the rate of pneumonia among patients with isolated chest trauma.
\end{abstract}

Crown Copyright (c) 2016 Published by Elsevier Ltd on behalf of College of Emergency Nursing Australasia. This is an open access article under the CC BY-NC-ND license (http://creativecommons.org/ licenses/by-nc-nd/4.0/).

\section{Introduction}

Failure to treat blunt chest injuries in a timely manner with sufficient analgesia, physiotherapy and respiratory support, often results in complications such as pneumonia and respiratory failure. These complications may cause long-term pulmonary impairment or death, delayed recovery and significantly increased resource use $[1,2]$. Rib fractures are reported to be the most common clinical fracture in older people ( $\geq 65$ years of age) [3] and this demographic is the most at risk of rib-fracture-related morbidity [1,4-6]. Patients with at least three rib fractures have a significantly increased risk

\footnotetext{
* Corresponding author at: Sydney Nursing School, The University of Sydney, 88 Mallett St, Camperdown, NSW 2006, Australia.

E-mail address: kate.curtis@sydney.edu.au (K. Curtis).
}

of in-hospital mortality [7], an effect even more pronounced in older patients in whom each additional rib fracture increases the risk of mortality by $19 \%$ and of pneumonia by $27 \%$ [5]. Even an isolated rib fracture is associated with significant consequences, particularly in the elderly [6]. Inadequate or delayed pain relief may cause anorexia, poor sleep, psychological stress and restricted movement, with inability to participate in normal activities [8]. Isolated blunt chest injury may result from a low energy mechanism (eg fall from standing), thus patients may not receive the rapid multidisciplinary response associated with a trauma team activation. The literature recommends implementing strategies such as clinical practice guidelines (herein pathways) to improve the care and outcomes of these patients [2,7]. However, pathways reported in the literature do not consider patients with less than three rib frac- 
tures, the elderly, or the burden of co-morbid disease, all of which are risk factors for morbidity and mortality $[2,9,10]$.

Despite improvements in pain management strategies in the emergency department, such as nurse-initiated analgesia, patients with moderate to severe pain are often left waiting for pain relief for longer than $60 \mathrm{~min}$ [11]. Evidence also shows that patients aged 75 years and older with pain-related Emergency Department (ED) visits are less likely to receive pain medication than younger patients [12]. Given that pain caused by blunt chest injury is associated with restricted pulmonary function which can lead to serious complications, the need for an effective early intervention in this patient group is critical.

To address this evidence-practice gap the trauma and emergency departments in our Level 1 Trauma Centre, in conjunction with the pain, physiotherapy and aged care teams developed and implemented a Chest Injury Protocol (ChIP) consolidating the best evidence available on treatment of blunt chest injury (Fig. 1). Comparable to a trauma team call or "stroke page", [13] which are known to improve patient and health service outcomes, ChIP is an early activation protocol. Early activation enables tailored, targeted patient care, as each patient has individual needs dependent on their pre-morbid condition. The intent was to facilitate multidisciplinary management of blunt chest trauma and effective multimodal analgesia to prevent respiratory compromise.

The aim of this study was to determine if the implementation of ChIP improved clinical outcomes and the delivery of healthcare services. We hypothesised that patients with blunt chest trauma who received a ChIP call would have reduced complications, improved health service delivery and shorter hospital length of stay (LOS) compared to similar patients who did not receive a ChIP call.

\section{Methods}

This was a retrospective before-after cohort study undertaken at St George Hospital, a 600-bed Level 1 Trauma Centre in Sydney, Australia, between August 2010 and November 2014. The study adhered to the National Statement on the Conduct of Human Research by the National Health and Medical Research Council of Australia and was approved by the South Eastern Sydney Local Health District Human Research Ethics Committee. In a previous study in patients with blunt thoracic trauma at St George Hospital the complication rate was $24 \%$ [14]. With $80 \%$ power to detect a minimum clinically important reduction in complications of $10 \%$, at an alpha level of 0.05 , a minimum of 240 participants were required in each arm of the study.

\section{The intervention}

Each member of the ChIP team (or their after-hours delegate) received a message via their personal pager and was required to respond within $60 \mathrm{~min}$. ChIP could be activated $24 \mathrm{~h}$ a day, 7 days a week by emergency nursing or medical staff. Initial intervention included incentive spirometry, high flow nasal prong oxygen (HFNP), multimodal analgesia including patient controlled analgesia (PCA) as indicated. Patients were then admitted to an appropriate acuity ward under the trauma service, or general surgical team after hours with transfer to trauma the following day. The trauma service coordinated the multidisciplinary care, involving specialty teams such as intensive care, cardiothoracic surgery and aged care as required [15]. Following the introduction of ChIP, All patients that received a ChIP call were entered into the trauma registry.

\section{Participants}

Patients were eligible for inclusion if they were aged 18 years or older and admitted to the study site with isolated blunt chest trauma, for example rib or sternal fractures, chest wall contusions

\section{Exclusion criteria}

Patients who received a trauma call were excluded as they receive the rapid multidisciplinary response associated with trauma team activation. Individuals who were intubated in the emergency department, had an Abbreviated Injury Scale (AIS) score greater than two in the head, neck, pelvis, and/or abdomen, or had extremity trauma requiring operative intervention were also excluded. The outcomes of those patients were likely to be influenced by the other major injuries [16] or the need for operative procedures.

For the before-ChIP cohort (August 2010-April 2012) potential patients were identified by searching the hospital clinical information database using International Classification of Diseases (ICD-10) codes related to thoracic trauma. This was cross-referenced with data from the hospital trauma registry using the thoracic AIS codes. The after-ChIP cohort comprised patients admitted from May 2012 to November 2014. A three week 'run in period', between protocol introduction and study inclusion allowed for implementation training and staff adjustment to the protocol. Patients that received a ChIP call were identified from the trauma registry.

\section{Data collection}

Medical records were reviewed for inclusion and exclusion criteria. A standardised template supported by a data dictionary was used for data extraction. Ninety-two records were not located (69 in the before group, 23 in the after group). Demographic information collected included age, gender and smoking habit. Smoking habit was recorded as current smoker or not a current smoker. We could not reliably distinguish smoking habit as current, past or never due to ambiguity of the frequently used term 'non-smoker' which could be valid for either a never or a past smoker. Clinical data collected included Injury Severity Score (ISS), new Injury Severity Score (nISS), AIS score, number of radiological rib fractures, time from injury to arrival, mechanism of injury, radiological evidence of a pneumothorax, haemothorax or pulmonary contusion within $24 \mathrm{~h}$ of injury, insertion of a tube thoracostomy, and the Charlson Comorbidity Index [17]. This Index is used to measure the burden of comorbid illness. A score $\geq 5$ is considered severe and indicates a high risk of dying from comorbid illness within one year [17]. Radiologic data were obtained from reports in the radiology database. AIS, ISS and nISS were hand scored by a trained AIS coder. A list of all included patients was provided to the hospital Casemix (finance) department to obtain hospital LOS data.

\section{Outcome measures}

The primary outcome was pneumonia. This was defined as radiological evidence of pulmonary air-space opacification, together with medical record documentation of a clinical diagnosis of pneumonia and treatment with antibiotics [2]. When radiological evidence of pulmonary air-space opacification developed within $24 \mathrm{~h}$ of hospital arrival, these changes were considered to represent contusion rather than infection. Secondary outcomes were divided into clinical and health service categories. Clinical outcomes were all-cause mortality, deep vein thrombosis, pulmonary embolism and the need for ventilatory support following admission (continuous or bi-level positive airway pressure, or endotracheal intubation). Health service outcome measures were hospital LOS, 


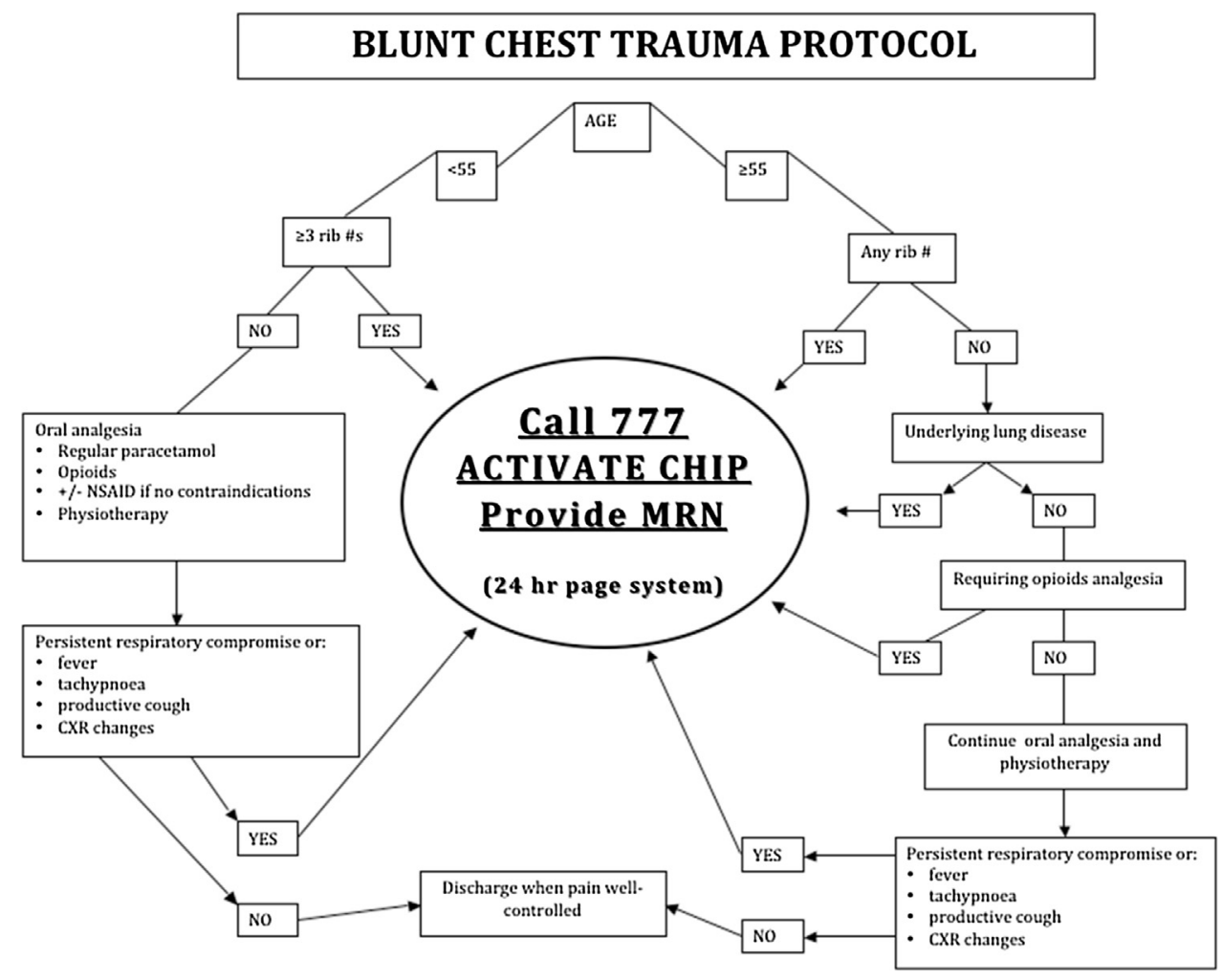

Fig. 1. ChIP: Blunt chest injury protocol.

NSAID: Non-steroidal anti-inflammatory drug; ChIP: Chest Injury Protocol; CXR: Chest X-ray; \#: fracture; MRN: Medical Record Number

time to pain team, physiotherapy and trauma team review and use of (PCA) and/or (HFNP).

\section{Data management and analysis}

Analysis was performed using IBM SPSS v21. Where data were missing, proportions were calculated using the total number of participants with a valid response as the denominator. The primary outcome of pneumonia was compared using logistic regression. The secondary outcome of hospital LOS was compared using multiple regression. Potential confounders considered for inclusion in the regression models were age, sex, ISS, Charlson Comorbidity Index, mechanism of injury, smoking history, tube thoracostomy, number of rib fractures, pulmonary contusion, pneumothorax and haemothorax. Initially, each potential confounding variable was examined separately (univariate analysis) to determine their association with the outcome variable. Confounders whose association with the outcome variable had a p value $<0.2$ were included in the multivariate model. A backward elimination approach was used for model reduction. A type I error rate of $\mathrm{p}<0.05$ was used for the test of hypotheses. The decision to retain a factor was based on its significance as well as the incremental amount of variance explained by the variable. Due to small counts in the remaining clinical outcomes of all-cause mortality, deep vein thrombosis, pulmonary embolism, and the need for ventilatory support, adjusted analyses for these outcomes were not attempted. Proportions were compared using Fischer's exact test where appropriate.

The outcomes of time to pain team and physiotherapy review, proportion of patients receiving pain team, trauma team and physiotherapy review, and use of PCA and/or HFNP oxygen were compared using the chi-square test or Fischer's exact test for proportions, and the Mann-Whitney $U$ test for continuous data (continuous data was all non-normally distributed). These outcomes were all components of ChIP and were presented to assess the effectiveness of protocol implementation. Therefore, no adjusted analyses were performed.

\section{Results}

There were 546 participants included in the study, 273 in the before-ChIP cohort and 273 in the after-ChIP cohort. Overall, the study cohort was an elderly population (median age of the beforeChIP cohort was 82 years (interquartile range: $71-88$ ) and of the after-ChIP cohort was 81 years (interquartile range: $70-87$ )) with falls from standing the most common cause of injury. The characteristics of participants in each group are presented in Table 1. Participants were similar in age, co-morbid status, smoking history, injury mechanism and chest injury severity. Participants in the after-ChIP cohort had a higher ISS and nISS. The univariate associations between potential confounding variables and the outcomes of pneumonia and hospital LOS are presented in Table 2.

Compared to those in the before-ChIP cohort, participants in the after-ChIP cohort were more likely to be reviewed by the pain team $(p<0.001)$, physiotherapy $(p=0.01)$, and the trauma team $(p<0.001)$. They were also more likely to receive HFNP oxygen $(\mathrm{p}<0.001)$ and PCA $(\mathrm{p}=0.04)$. Physiotherapy review occurred $4 \mathrm{~h}$ earlier in the after-Chip cohort $(p<0.001)$ (Table 3$)$.

Twenty five participants (9.2\%) in the before-ChIP cohort developed pneumonia compared with $12(4.4 \%)$ in the after-ChIP cohort, a reduction of $4.8 \%(95 \% \mathrm{CI} 0.5-9.2, \mathrm{p}=0.03)$. After adjusting for confounding variables, this difference remained significant with the odds of a participant developing pneumonia in the after-ChIP cohort 56\% lower than before ChIP introduction (OR 0.44, 95\% CI $0.21-0.90, \mathrm{p}=0.03)$. Six participants $(2.2 \%)$ in the before-ChIP cohort required ventilatory support, compared to three patients (1.1\%) in the after-ChIP cohort, a difference of $1.1 \%$ (95\% CI -1.3-3.7, $\mathrm{p}=0.50)$. Six participants $(2.2 \%)$ in the before-ChIP cohort died, compared to two $(0.7 \%)$ in the after-ChIP cohort, a difference of 
Table 1

Comparison of patient characteristics in the before-ChIP and after-ChIP cohorts.

\begin{tabular}{|c|c|c|c|c|c|}
\hline \multirow[t]{2}{*}{ Characteristics } & \multicolumn{2}{|c|}{ Before-ChIP Cohort $n=273$} & \multicolumn{2}{|c|}{ After-ChIP Cohort $n=273$} & \multirow[t]{2}{*}{$\mathrm{p}$ value } \\
\hline & Median & IQR & Median & IQR & \\
\hline Age (years) & 82 & $71-88$ & 81 & $70-87$ & 0.73 \\
\hline ISS & 3 & $2-9$ & 5 & $2-9$ & $<0.001$ \\
\hline nISS & 3 & $2-9$ & 5 & $2-9$ & $<0.001$ \\
\hline AIS score chest & 1 & $1-2$ & 1 & $1-3$ & 0.39 \\
\hline Number of radiological rib fractures & 0 & $0-2$ & 0 & $0-3$ & 0.42 \\
\hline Time from injury to arrival (hours) & 3.1 & $1.2-26.3$ & 8.1 & $1.4-45.6$ & 0.12 \\
\hline \multirow[t]{2}{*}{ Charlson Co-Morbidity Score } & 1 & $0-2$ & 1 & $0-2$ & 0.15 \\
\hline & $\mathrm{n}(\%)$ & $95 \% \mathrm{CI}$ & $\mathrm{n}(\%)$ & $95 \% \mathrm{CI}$ & p value \\
\hline Charlson Score $\geq 5$ & $4(1.5)$ & $0.1-2.9$ & $7(2.6)$ & $0.7-4.4$ & 0.36 \\
\hline Male & $119(43.6)$ & $37.7-49.5$ & $129(47.3)$ & $41.3-53.2$ & 0.39 \\
\hline Current smoker & $35(14.5)$ & $10.3-18.7$ & $31(13.1)$ & $9.1-17.1$ & 0.66 \\
\hline Mechanism of injury: & - & - & - & - & 0.16 \\
\hline Motor vehicle collision & $17(6.2)$ & $3.3-9.1$ & $8(2.9)$ & $0.9-4.9$ & - \\
\hline Vulnerable road user & $6(2.2)$ & $0.5-3.9$ & $5(1.8)$ & $0.2-3.4$ & - \\
\hline Fall $<1 \mathrm{~m}$ & $224(82.1)$ & $77.5-86.6$ & $234(85.7)$ & $81.6-89.9$ & - \\
\hline Fall $>1 \mathrm{~m}$ & $20(7.3)$ & $4.2-10.4$ & $14(5.1)$ & $2.5-7.7$ & - \\
\hline Other & $6(2.2)$ & $0.5-3.9$ & $12(4.4)$ & $2.0-6.8$ & - \\
\hline Haemothorax & $27(9.9)$ & $6.3-13.4$ & $29(10.6)$ & $7.0-14.3$ & 0.78 \\
\hline Pneumothorax & $16(5.9)$ & $3.1-8.6$ & $10(3.7)$ & $1.4-5.9$ & 0.23 \\
\hline Pulmonary contusion & $4(1.5)$ & $0.0-2.9$ & $2(0.7)$ & $-0.3-1.7$ & 0.69 \\
\hline Tube thoracostomy performed & $8(2.9)$ & $0.9-4.9$ & $8(2.9)$ & $0.9-4.9$ & 1.00 \\
\hline
\end{tabular}

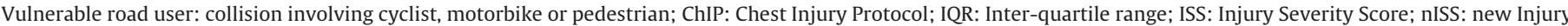
Severity Score; AIS: Abbreviated Injury Scale; CI: Confidence Interval.

Table 2

Univariate analysis of the association with the outcome variables.

\begin{tabular}{|c|c|c|c|c|}
\hline Outcome & Category & OR & $95 \% \mathrm{CI}$ & p value \\
\hline \multirow[t]{12}{*}{ Pneumonia } & Age (per 1 year increase) & 1.024 & $0.996-1.053$ & 0.091 \\
\hline & Sex (male) & 1.023 & $0.524-1.998$ & 0.947 \\
\hline & ISS (per 1 unit increase) & 1.027 & $0.956-1.104$ & 0.469 \\
\hline & Current Smoker & 1.166 & $0.432-3.143$ & 0.762 \\
\hline & Mechanism of Injury & & & 0.805 \\
\hline & Time from injury to arrival (hours) & 1.000 & $0.999-1.000$ & 0.629 \\
\hline & Charlson Co-Morbidity Index (per 1 unit increase) & 1.218 & $0.983-1.508$ & 0.071 \\
\hline & Number of rib fractures (per 1 unit increase) & 1.136 & $0.988-1.307$ & 0.074 \\
\hline & Presence of pulmonary contusion & 2.800 & $0.319-24.609$ & 0.353 \\
\hline & Presence of pneumothorax & 1.864 & $0.533-6.523$ & 0.330 \\
\hline & Presence of haemothorax & 4.362 & $2.023-9.408$ & $<0.001$ \\
\hline & Tube thoracostomy performed & 3.367 & $0.915-12.385$ & 0.068 \\
\hline Outcome & Category & Test Statistics & $\mathrm{p}$ value & \\
\hline \multirow[t]{12}{*}{ Hospital LOS } & Age (per 1 year increase) & $r=0.219$ & $<0.001$ & \\
\hline & Sex (male) & $\mathrm{U}=31050.000$ & 0.001 & \\
\hline & ISS (per 1 unit increase) & $r=0.251$ & $<0.001$ & \\
\hline & Current Smoker & $U=13277.500$ & 0.782 & \\
\hline & Mechanism of Injury & $U=29.329$ & $<0.001$ & \\
\hline & Time from injury to arrival & $r=-0.051$ & 0.239 & \\
\hline & Charlson Co-Morbidity Index (per 1 unit increase) & $r=0.140$ & 0.001 & \\
\hline & Number of rib fractures (per 1 unit increase) & $r=0.182$ & $<0.001$ & \\
\hline & Presence of pulmonary contusion & $\mathrm{U}=1212.500$ & 0.286 & \\
\hline & Presence of pneumothorax & $\mathrm{U}=5466.000$ & 0.097 & \\
\hline & Presence of haemothorax & $\mathrm{U}=8935.000$ & $<0.001$ & \\
\hline & Tube thoracostomy performed & $U=1856.500$ & $<0.001$ & \\
\hline
\end{tabular}

OR: Odds Ratio.

Table 3

Hospital treatment characteristics in the before-ChIP and after-ChIP cohorts.

\begin{tabular}{|c|c|c|c|}
\hline Characteristic & Before-ChIP Cohort $n=273$ & After-ChIP Cohort $\mathrm{n}=273$ & $\mathrm{p}$ value \\
\hline Pain Team Review, n (\%, 95\% CI) & $36(13.2,8.9-17.5)$ & $87(31.9,26-37.8)$ & $<0.001$ \\
\hline Median time to pain team review (hours) (IQR) & $30(20-45)$ & $27(19-45)$ & 0.77 \\
\hline Physiotherapy review, n (\%, 95\% CI) & $235(86.1,81.7-90.5)$ & $255(93.4,90.3-96.5)$ & 0.01 \\
\hline Median time to physiotherapy review (hours) (IQR) & $24(18-41)$ & $20(7-28)$ & $<0.001$ \\
\hline Trauma team review, $\mathrm{n}(\%, 95 \% \mathrm{CI})$ & $107(39.2,33-45.4)$ & $258(94.5,91.6-97.4)$ & $<0.001$ \\
\hline PCA used, $\mathrm{n}(\%, 95 \% \mathrm{CI})$ & $28(10.3,6.4-14.1)$ & $44(16.1,11.5-20.8)$ & 0.04 \\
\hline HFNP used, $\mathrm{n}(\%, 95 \% \mathrm{CI})$ & $28(10.3,6.4-14.1)$ & $116(42.5,36.2-48.7)$ & $<0.001$ \\
\hline
\end{tabular}

ChIP: Chest Injury Protocol; CI: Confidence Interval; IQR: Inter-quartile range; PCA: Patient Controlled Analgesia; HFNP: High flow nasal prong oxygen. 
Table 4

Patient and hospital outcomes in the before-ChIP and after-ChIP cohorts.

\begin{tabular}{|c|c|c|c|}
\hline \multirow[t]{2}{*}{ Characteristic } & \multirow{2}{*}{$\frac{\text { Before-ChIP Cohort n }=273}{\text { Median (IQR) }}$} & \multirow{2}{*}{$\begin{array}{l}\text { After-ChIP Cohort } \mathrm{n}=273 \\
\text { Median (IQR) }\end{array}$} & \multirow[t]{2}{*}{$\mathrm{p}$ value } \\
\hline & & & \\
\hline Hospital LOS (days) & $\begin{array}{l}4(2-8) \\
n(\%, 95 \% C I)\end{array}$ & $\begin{array}{l}4(2-8) \\
\mathrm{n}(\%, 95 \% \mathrm{CI})\end{array}$ & 0.50 \\
\hline Pneumonia & $25(9.2,5.5-12.8)$ & $12(4.4,1.8-7.0)$ & 0.03 \\
\hline DVT & 0 & 0 & - \\
\hline $\mathrm{PE}$ & 0 & 0 & - \\
\hline Ventilator Support Required (CPAP/BiPAP/intubation) & $6(2.2,0.3-4.0)$ & $3(1.1,-0.2-2.4)$ & $0.50^{*}$ \\
\hline Death & $6(2.2,0.3-4.0)$ & $2(0.7,-0.3-1.8)$ & $0.30^{*}$ \\
\hline
\end{tabular}

" Fischer's exact test used ChIP: Chest Injury Protocol; IQR: Inter-quartile range; LOS: Length of stay; DVT: Deep venous thrombosis; PE: Pulmonary embolism; CPAP: Continuous positive airway pressure; BiPAP: Bi-level positive airway pressure.

$1.5 \%$ (95\% CI $-0.8-4.0, \mathrm{p}=0.29$ ). No deep vein thromboses or pulmonary emboli were recorded in either cohort. The unadjusted patient outcome results are presented in Table 4. The median hospital LOS remained the same ( 4 days in both cohorts, $p=0.50$ ). Even after adjusting for confounding variables, no significant difference in hospital LOS between the cohorts existed (adjusted mean difference in LOS -0.2 days, $95 \% \mathrm{CI}-1.2-0.8, \mathrm{p}=0.74)$.

\section{Discussion}

This retrospective cohort study evaluated the impact of facilitated multidisciplinary management, via an early activation system, on outcomes and healthcare delivery amongst patients with blunt chest injury. The introduction of ChIP was associated with a decrease in the incidence of pneumonia with the number needed to treat to prevent one case of pneumonia being 21 patients. There were also trends toward a lower incidence of death and ventilatory support, although a much larger study would be required to demonstrate statistically significant differences for these outcomes. This reduction in pneumonia could be attributed to the more efficient and enhanced delivery of healthcare services, such as trauma team review, physiotherapy and pain team review. The results do not support the hypothesis, that ChIP would result in shorter hospital LOS through providing patients with improved oxygen delivery, physiotherapy and pain relief. The after-CHiP cohort had a higher median ISS which may explain the lack of effect of ChIP on LOS, though it should be noted that both cohorts fall into the minor injury category. In addition, LOS is a result of multiple factors which may not depend on injury severity or management especially in the elderly.

There are several limitations with this study which the following two paragraphs address. Data were collected retrospectively from the medical record, so the integrity of the data is dependent upon the accuracy with which the data was recorded. Differences in outcomes measured in a before and after study design are open to confounding due to differences in outcomes that occur over time regardless of the study intervention. While we have attempted to adjust for confounding in our analysis, there may still be other unmeasured variables that have not been accounted for. In evaluating a before/after study, it is important to determine whether patients in the before cohort would have been eligible for inclusion in the after cohort, which was the case with this study.

Measurement bias for the diagnosis of pneumonia may have occurred and the diagnosis of pneumonia misclassified, as it was determined based on retrospective evidence from the medical records, radiological imaging and medication charts. Difficulties exist in defining pneumonia in a retrospective study. The strict definition by the Centre for Disease Control incorporates radiological evidence, clinical evidence of fever or hypothermia, leucopenia or leukocytosis and bacteriological confirmation. This definition would have missed most pneumonia cases in this study as bacteriological confirmation by blood or sputum culture is rarely done in this clinical setting [18]. To improve the sensitivity of detection, we chose a pragmatic definition of pneumonia that was more reflective of clinical practice. This was a single-centre study conducted in a level 1 trauma centre so it may not be appropriate to generalize the results to other healthcare settings. A cluster randomized trial across different hospital types (for example metropolitan, rural and regional) would provide more robust evidence and determine external validity. A larger prospective study would also enable factor analysis of the individual treatments (analgesia, chest physiotherapy and high flow nasal prong oxygen), to determine which particular patient groups would benefit from particular interventions.

Despite these limitations, ChIP represents a considerable improvement on previous management approaches for blunt chest trauma at our hospital. Prior to ChIP, clinicians from a variety of medical specialties including respiratory, aged care and cardiothoracic surgery were involved in the inpatient care of these patients. This led to marked differences in management. ChIP ensured that all patients were admitted under a consultant-led trauma team, creating a consistent approach to the management of blunt chest trauma. A trauma case management model of care allowed the patient to be followed throughout their hospital stay by a specialist trauma nurse [19] who was integral to facilitating multidisciplinary review and reinforced compliance with the early activation and "care bundle". Care was organised through multidisciplinary trauma meetings, where medical, surgical and allied health professionals met to discuss care. These are known to decrease hospital LOS and streamline the care of trauma patients [20].

Compared to other blunt chest trauma populations reported in the literature, there was a higher proportion of patients injured from a low level fall in our study, as most patients in motor vehicle collisions, high-level falls and assaults would have met trauma-call criteria and were excluded [10]. Our population was also older and had a greater burden of comorbid disease than other blunt chest trauma populations in the literature [4]. The findings of our study support the implementation of ChIP to coordinate care to benefit all patients with blunt chest trauma, rather than only those with a certain number of rib fractures. Given the demographic of participants in this study, this intervention is particularly relevant to the care of elderly patient with blunt chest trauma. It has been reported that $50 \%$ of rib fractures cannot be detected by plain antero-posterior chest $\mathrm{x}$-rays, and diagnosis of rib fractures often relies on clinical judgment [21]. Further, it highlights the importance of incorporating evidence around risk factors for complications into clinical pathways, for example underlying respiratory disease, age and cardiopulmonary disease.

Identification of the after-ChIP cohort was from the trauma registry, and thus reliant upon ChIP uptake and knowledge by staff to actually activate a ChIP call. It is unknown if there were any patients eligible for ChIP who did not receive a call. Implementation barriers may have prevented less injured, but qualifying patients, from receiving a ChIP page, which may account for the difference in ISS 
and nISS between the two cohorts. Additional investigation into the enablers and barriers of implementation is recommended. Barriers to implementation in evidence-based care include lack of time, lack of resources, poor access to guidelines, a lack of continuing education and preconceived opinions [22]. These barriers could affect the type of patients that receive ChIP, the timeliness of interventions and the delivery of all services. While ChIP is a sustainable intervention requiring no additional staffing or financial resources it does increase the workload of the pain team, trauma team and physiotherapists, which may influence staff opinion and use. Emergency and ward nursing staff were required to upskill in HFNP oxygen application, and an elearning program was subsequently developed to facilitate this [23].

ChIP enabled a personalised medicine approach to tailor treatment to the patient's individual characteristics ${ }^{24}$ which can overcome some of the inflexibility and limitations of clinical pathways [24]. While not the original intent, it also resulted in a blunt chest injury "care bundle", or, a uniform set of evidence based practices specific to a clinical presentation to be considered by the attending team [25]. Although the individual components of this bundle, comprising humidified high-flow nasal prong oxygen, physiotherapy, analgesia and trauma care coordination were not analysed separately in this study, each intervention has been well supported in the literature for improving outcomes in patients with blunt chest trauma [26]. Further refinement and evaluation of ChIP could validate and formalise the blunt chest injury "care bundle".

\section{Conclusion}

The ChIP intervention is an evidence-informed early activation protocol introduced to expedite care of patients with blunt chest trauma. ChIP reduced the incidence of pneumonia and improved the delivery of healthcare services.

\section{Funding}

Author Curtis is supported by a Translation of Research into Practice grant (GNT1067639_Curtis, 2013-15) from the National Health and Medical Research Council. The purchase of equipment for this study was funded by a Motor Accidents Authority Small Grant (now known as State Insurance Regulatory Authority). The funding sources had no involvement in the conduct of the research and/or preparation of the article.

\section{Provenance and conflicts of interest}

Author Curtis is Associate Editor (Trauma) with the Australasian Emergency Nursing Journal but had no role to play with the peer review or editorial decision-making of this manuscript whatsoever.

\section{Statement of contributorship}

KC, SA conceived and designed the study. KC and SA oversaw all aspects of the study. KC, SA, AU and HG conducted data acquisition. MLang and DD devised the intervention. KC oversaw implementation of the intervention. AU and MLam conducted data analysis. KC, SA, AU and MLam interpreted the data. All authors have been actively involved in the drafting and critical revision of the manuscript.

\section{References}

[1] Sharma OP, Oswanski MF, Jolly S, Lauer SK, Dressel R, Stombaugh HA. Perils of rib fractures. Am Surg 2008;74:310-4.

[2] Todd SR, McNally MM, Holcomb JB, Kozar RA, Kao LS, Gonzalex EA, et al. A multidisciplinary clinical pathway decreases rib fracture-associated infectious morbidity and mortality in high-risk trauma patients. Am J Surg 2006;192:806-11.

[3] Barrett-Connor E, Nielson CM, Orwoll E, Bauer DC, Cauley JA. (2010). Epidemiology of rib fractures in older men: Osteoporotic Fractures in Men (MrOS) prospective cohort study. BMJ, 340 doi:10.1136/bmj.c1069.

[4] Bergeron E, Lavoie A, Clas D, Moore L, Rattle S, Tetreault S, et al. Elderly trauma patients with rib fractures are at greater risk of death and pneumonia. J Trauma 2003;54:478-85.

[5] Bulger EM, Arneson MA, Mock CN, Jurkovich GJ. Rib fractures in the elderly. J Trauma 2000;48:1040-6.

[6] Elmistekawy E, Hammad AA. Isolated rib fractures in geriatric patients. Ann Thorac Med 2007;2:166-8.

[7] Testerman GM. Adverse outcomes in younger rib fracture patients. South Med J 2006;99:335-9.

[8] Møiniche S, Kehlet H, Dahl JB. A qualitative and quantitative systematic review of preemptive analgesia for postoperative pain relief: the role of timing of analgesia. Anesthesiology 2002;96:725-41.

[9] Sesperez J, Wilson S, Jalaludin B, Seger M, Sugrue M. Trauma case management and clinical pathways: prospective evaluation of their effect on selected patient outcomes in five key trauma conditions. J Trauma 2001:50:643-9.

[10] Sahr SM, Webb ML, Renner CH, Sokol RK, Swegle JR. Implementation of a rib fracture triage protocol in elderly trauma patients. J Trauma Nurs 2013;20:172-5.

[11] Shaban RZ, Holzhauser K, Gillespie K, Huckson S, Bennetts S. Characteristics of effective interventions supporting quality pain management in Australian emergency departments: an exploratory study. Australas Emerg Nurs J 2012;15:23-30.

[12] Platts-Mills TF, Esserman DA, Brown DL, Bortsov AV, Sloane PD, McLean SA Older US emergency department patients are less likely to receive pain medication than younger patients: results from a national survey. Ann Emerg Med 2012;60:199-206.

[13] Batmanian JJ, Lam M, Matthews C, Finckh A, Duffy M, Wright R, et al. A protocol-driven model for the rapid initiation of stroke thrombolysis in the emergency department. Med J Aust 2007;187:567-70.

[14] Asha S, Curtis K, Taylor C, Kwok A. Patient-controlled analgesia compared with interval analgesic dosing for reducing complications in blunt thoracic trauma: a retrospective cohort study. Emerg Med J 2013;30(December):1024-8.

[15] Ursic C, Curtis K, Zou Y, Black D. Improved trauma patient outcomes after implementation of a dedicated trauma admitting service. Injury 2009;40:99-103.

[16] Baker SP, o'Neill B, Haddon W, Long WB. The injury severity score: a method for describing patients with multiple injuries and evaluating emergency care. J Trauma 1974;14:187-96.

[17] Charlson ME, Pompei P, Ales KL, MacKenzie CR. A new method of classifying prognostic comorbidity in longitudinal studies: development and validation. J Chronic Dis 1987;40:373-83.

[18] Horan TC, Andrus M, and Dudeck MA. (2008). CDC/NHSN surveillance definition of health care-associated infection and criteria for specific types of infections in the acute care setting. Am J Infect Control, 36:309-332.

[19] Curtis K, Zou Y, Morris R, Black D. Trauma case management: improving patient outcomes. Injury 2006;37:626-32.

[20] Dutton RP, Cooper C, Jones A, Leone S, Kramer ME, Scalea TM. Daily multidisciplinary rounds shorten length of stay for trauma patients. J Trauma 2003;55:913-9.

[21] Bansidhar BJ, Lagares-Garcia JA, Miller SL. Clinical rib fractures: are follow-up chest X-rays a waste of resources. Am Surg 2002;68:449-53.

[22] Wallis L. Barriers to implementing evidence-based practice remain high for U.S. nurses. Am J Nurs 2012;112:15.

[23] Curtis K, Wiseman T, Kennedy B, Kourouche S, Goldsmith H. Implementation and evaluation of a ward-based eLearning program for trauma patient management. J Trauma Nurs 2016;23:28-35.

[24] Goldberger JJ, Buxton AE. Personalized medicine vs guideline-based medicine. JAMA 2013;309:2559-60.

[25] Resar R, Pronovost P, Haraden C, Simmonds T, Rainey T, Nolan T. Using a bundle approach to improve ventilator care processes and reduce ventilator-associated pneumonia. Jt Comm J Qual Patient Saf 2005;31:243-8.

[26] Unsworth A, Curtis K, Asha S. Treatments for blunt chest trauma and their impact on patient outcomes and health service delivery. Scand J Trauma Resusc Emerg Med 2015;23:17. 